\title{
USING A CELL FLUID MODEL FOR THE DESCRIPTION OF A PHASE TRANSITION IN SIMPLE LIQUID ALKALI METALS
}

\begin{abstract}
This article embraces a theoretical description of the first-order phase transition in liquid metals with the application of a cell fluid model. The results are obtained through the calculation of the grand partition function without a usage of phenomenological parameters. The Morse potential is used for the calculation of the equation of state and the coexistence curve. Specific results for sodium and potassium are obtained. Comparison of the outcome of analytic expressions with data of computer simulations is presented.

Keywords: cell fluid model, coexistence curve, collective variables, equation of state, firstorder phase transition.
\end{abstract}

\section{Introduction}

This article is based on the method we proposed in [8]. It enables one to obtain the equation of state of the cell model in a wide range of temperatures below and above the critical point. Particular analytic results were conducted with the use of the Morse potential

$U(r)=\epsilon e^{-2\left(r-R_{0}\right) / \alpha}-2 \epsilon e^{-\left(r-R_{0}\right) / \alpha}$.

The consequence of the approach $[8,10]$ is a restriction of the ratio between the coordinate of minimum $R_{0}$ and the effective reach $\alpha$ of the interaction potential $R_{0} / \alpha<4 \ln 2$. However, according to numerical results $[4,12]$, this ratio exceeds $R_{0} / \alpha=4 \ln 2$ for real substances, in particular, for fluid metals. In the present article, the method proposed in [8] and slightly changed in [10] is modified by means of introducing a temperature-free effective interaction potential. This makes it applicable in the range $R_{0} / \alpha>$ $4 \ln 2$ for a description of real metals in the region of a first-order phase transition.

This paper is laid as follows: in Section 2, the temperature-free effective interaction potential is introduced, and the main steps of calculations toward obtaining an exact representation of the grand partition function of the cell fluid model are shown. This expression is restricted to $\rho^{4}$-model and calculated in the mean-field approximation in Section 3. Section 4

(c) M.P. KOZLOVSKII, O.A. DOBUSH, I.V. PYLYUK, 2017

ISSN 2071-0194. Ukr. J. Phys. 2017. Vol. 62, No. 10 is devoted to an equation of state of the cell fluid applicable in a wide temperature region except for a vicinity of the critical point. In Section 4 , the analytic result obtained in this work is compared with simulation data [15] for parameters of the Morse potential describing alkali metals $N a$ and $K$. The discussion and conclusions are presented in Section 5 .

\section{Representation of the Grand Partition Function}

The objective of our investigation is the description of the behavior of a simple fluid in a wide temperature region. For this purpose within the grand canonical ensemble, we will calculate the grand partition function (GPF) of the cell fluid model as an approximation of the real continuous system and obtain the result in the form of a function of the temperature and the density.

The idea of the cell fluid $[1,2]$ consists in a fixed partition of system's volume $V$, where $N$ particles reside, on $N_{v}$ mutually disjoint elementary cubes, each of volume $v=V / N_{v}$. In a formalism of the cell model, the GPF of a system of volume $V$ with $N$ particles takes the form

$\Xi=\sum_{N=0}^{\infty} \frac{(z)^{N}}{N !} \int_{V}(d x)^{N} \exp \left[-\frac{\beta}{2} \sum_{\mathbf{j}_{1}, \mathbf{j}_{2} \in \Lambda} \tilde{U}_{j_{12}} \rho_{\mathbf{j}_{1}}(\eta) \rho_{\mathbf{j}_{2}}(\eta)\right]$ 
Here, $z=e^{\beta \mu}$ is the activity, $\beta$ is the inverse temperature, and $\mu$ is the chemical potential. In this expression, $\int_{V}(d x)^{N}=\int_{V} d x_{1} \ldots \int_{V} d x_{N}$ denotes the integration over the coordinates $x_{i}=\left(x_{i}^{(1)}, x_{i}^{(2)}, x_{i}^{(3)}\right)$ of all particles in the system, $\eta=\left\{x_{1}, \ldots, x_{N}\right\}$ is the set of coordinates, and $j_{12}=\left|\mathbf{j}_{1}-\mathbf{j}_{2}\right|$ is the difference between two cell vectors. The vectors $\mathbf{j}_{1}$ and $\mathbf{j}_{2}$ take on values from the set $\Upsilon$ defined as

$\Upsilon=\left\{\mathbf{j}=\left(j_{1}, j_{2}, j_{3}\right) \mid j_{i}=c m_{i} ; m_{i}=1,2, \ldots N_{a} ;\right.$

$\left.i=1,2,3 ; \quad N_{v}=N_{a}^{3}\right\}$.

Here, $c$ is the linear size of each cell, $N_{a}$ is the number of cells along each axis. Values $\rho_{\mathbf{j}}(\eta)$ are the occupation numbers of cells $[6,10,14]$. The interaction potential has the form

$\tilde{U}_{l_{12}}=-U_{l_{12}}+\Psi_{l_{12}}$

$\Psi_{l_{12}}=D e^{-2\left(l_{12}-1\right) / \alpha_{R}}, \quad U_{l_{12}}=2 D e^{-\left(l_{12}-1\right) / \alpha_{R}}$,

$l_{12}$ is the difference between two vectors $\mathbf{l}_{1}$ and $\mathbf{l}_{2}$ from the set

$\Lambda=\left\{\mathbf{l}=\left(l_{1}, l_{2}, l_{3}\right) \mid l_{i}=c m_{i} / R_{0} ; \quad m_{i}=1,2, \ldots, N_{a} ;\right.$

$\left.i=1,2,3 ; \quad N_{v}=N_{a}^{3}\right\}$.

Moreover, $l_{12}=j_{12} / R_{0}$. $R_{0}$ corresponds to the minimum of the function $\tilde{U}_{l_{12}}\left[\tilde{U}\left(l_{12}=1\right)=-D\right.$ is the potential well depth]. For the sake of convenience here and henceforth, we measure the length in $R_{0^{-}}$ units. Thus, $\alpha_{R}=\alpha / R_{0}$ is the effective interaction radius $\alpha$ in $R_{0}$-units.

Looking at (2.2), it becomes obvious that different particles in the same cell interact with each other equally and irrespective of the distance between them. The interaction between constituents of different cells is a function $\tilde{U}_{l_{12}}$ of the distance between cells.

As we had shown in [10] in terms of the Fourier representation, GPF (2.1) contains a sum of diagonal terms in the exponent. It can be expressed via $N$ integrals over the coordinates of particles and $N_{v}$ integrals over the collective variables $(\mathrm{CV}) \rho_{\mathbf{k}}$

$$
\begin{aligned}
& \Xi=\sum_{N=0}^{\infty} \frac{(z)^{N}}{N !} \int_{V}(d x)^{N} \exp \left[-\frac{\beta}{2} \sum_{\mathbf{k} \in \mathcal{B}_{c}} \tilde{U}(k) \hat{\rho}_{\mathbf{k}} \hat{\rho}_{-\mathbf{k}}\right] \times \\
& \times \int(d \rho)^{N_{v}} \int(d \nu)^{N_{v}} \exp \left[2 \pi i \sum_{\mathbf{k} \in \mathcal{B}_{c}} \nu_{\mathbf{k}}\left(\rho_{\mathbf{k}}-\hat{\rho}_{\mathbf{k}}\right)\right] .
\end{aligned}
$$

Herewith,

$(d \rho)^{N_{v}}=\prod_{\mathbf{k} \in \mathcal{B}_{c}} d \rho_{\mathbf{k}} ; \quad(d \nu)^{N_{v}}=\prod_{\mathbf{k} \in \mathcal{B}_{c}} d \nu_{\mathbf{k}}$.

The operator $\hat{\rho}_{\mathbf{k}}$ is the representation of the occupation number $\rho_{\mathbf{l}}(\eta)$ in the reciprocal space

$\hat{\rho}_{\mathbf{k}}=\frac{1}{\sqrt{N_{v}}} \sum_{\mathbf{l} \in \Lambda} \rho_{\mathbf{l}}(\eta) e^{i \mathbf{k} \mathbf{l}}$.

The vector $\mathbf{k}$ takes values from the set $\mathcal{B}_{c}$ corresponding to one cell

$\mathcal{B}_{c}=\left\{\mathbf{k}=\left(k_{1}, k_{2}, k_{3}\right) \mid k_{i}=-\frac{\pi}{c}+\frac{2 \pi}{c} \frac{n_{i}}{N_{1}}\right.$,

$\left.n_{i}=1,2, \ldots, N_{a} ; \quad i=1,2,3 ; \quad N_{v}=N_{a}^{3}\right\}$.

The Fourier transform of the Morse potential (2.2) $\tilde{U}(k)=-U(k)+\Psi(k)(k=|\mathbf{k}|)$ is as follows:

$U(k)=U(0)\left(1+\alpha_{R}^{2} k^{2}\right)^{-2}, \Psi(k)=\Psi(0)\left(1+\frac{\alpha_{R}^{2} k^{2}}{4}\right)^{-2}$.

$U(0)=16 D \pi \frac{\alpha_{R}^{3}}{v} e^{R_{0} / \alpha}, \Psi(0)=D \pi \frac{\alpha_{R}^{3}}{v} e^{2 R_{0} / \alpha}$.

Hence, $\chi$ is a real positive parameter $(\chi>0)$, which is fixed for each particular substance. $v=v / R_{0}^{3}$, $\beta_{c}=1 / k_{\mathrm{B}} T_{c}, k_{\mathrm{B}}$ is the Boltzman constant, $T_{c}$ is some fixed temperature, which will be defined later. Let us transfer a part of the repulsive interaction $\chi \Psi(0)>0$ from the initial interaction potential $\tilde{U}(k)>0$ to the Jacobian of the transition from individual coordinates to collective variables in order to write its accurate representation. The similar idea was used by us in [10]. Now instead of $\tilde{U}(k)$, we introduce the effective potential of interaction

$W(k)=U(k)-\Psi(k)+\chi \Psi(0)$.

It is easy to see that a sum of $\chi \Psi(0)$ and $-W(k)$ is equal to the initial potential of interaction (2.2).

The difference between (2.4) and an analogous expression in $[8,10]$ is that the present explicit expression of the effective potential of interaction is temperature-free. The GPF of the model in the representation of collective variables $\rho_{\mathbf{k}}$ has the following form:

$$
\begin{aligned}
& \Xi=\int(d \rho)^{N_{v}} \exp \left[\beta \mu \rho_{0}+\frac{\beta}{2} \sum_{\mathbf{k} \in \mathcal{B}_{c}} W(k) \rho_{\mathbf{k}} \rho_{-\mathbf{k}}\right] \times \\
& \times \prod_{l=1}^{N_{v}}\left[\sum_{m=0}^{\infty} \frac{v^{m}}{m !} e^{-p(T) m^{2}} \delta\left(\rho_{\mathbf{l}}-m\right)\right] .
\end{aligned}
$$


Note that $\rho_{\mathbf{l}}$ is the representation of $\rho_{\mathbf{k}}$ in the direct space, $l=|\mathbf{l}|$, and the parameter $p$ is a function of the temperature:

$p(T)=\chi \beta \Psi(0) / 2$,

which is different from an analogous temperature-free parameter in $[8,10]$. This complicates the calculation of GPF (2.5).

The second modification of the previously developed method $[7,8]$ is the application of the Stratonovich-Hubbard transformation to the term, which contains the effective potential of interaction

$\exp \left[\frac{\beta}{2} \sum_{\mathbf{k} \in \mathcal{B}_{c}} W(k) \rho_{\mathbf{k}} \rho_{-\mathbf{k}}\right]=$

$=g_{W} \int(d t)^{N_{v}} \exp \left[-\frac{1}{2 \beta} \sum_{\mathbf{k} \in \mathcal{B}_{c}} \frac{t_{\mathbf{k}} t_{-\mathbf{k}}}{W(k)}+\sum_{\mathbf{k} \in \mathcal{B}_{c}} t_{\mathbf{k}} \rho_{\mathbf{k}}\right]$.

Note that $W(k)>0$ for all $\chi>0$. We have

$g_{W}=\prod_{\mathbf{k} \in \mathcal{B}_{c}}(2 \pi \beta W(k))^{-1 / 2}$.

The variables $t_{\mathbf{k}}$ are complex values $t_{\mathbf{k}}=t_{\mathbf{k}}^{(c)}-i t_{\mathbf{k}}^{(s)}$, for which $t_{\mathbf{k}}^{(c)}$ and $t_{\mathbf{k}}^{(s)}$ are real and imaginary parts, respectively.

\section{Application of the Cumulant Representation}

When using the method of collective variables, it is convenient to represent the Jacobian of the transition $J\left(\rho_{\mathbf{l}}\right)$ as a cumulant expansion $[8,16]$

$\tilde{J}_{l}\left(\tilde{t}_{\mathbf{l}}\right)=\exp \left[-\sum_{n=0}^{\infty} \frac{a_{n}(T)}{n !} \rho_{\mathbf{1}}^{n}\right]$.

We calculated the functional form of cumulants $a_{n}(T)$ :

$$
\begin{aligned}
& a_{0}(T)=-\ln T_{0}(v, p(T)) ; \quad a_{1}(T)=-\frac{T_{1}(v, p(T))}{T_{0}(v, p(T))} \\
& a_{2}(T)=-\frac{T_{2}(v, p(T))}{T_{0}(v, p(T))}+a_{1}^{2} \\
& a_{3}(T)=-\frac{T_{3}(v, p(T))}{T_{0}(v, p(T))}-a_{1}^{3}(T)+3 a_{1}(T) a_{2}(T) ; \\
& a_{4}(T)=-\frac{T_{4}(v, p(T))}{T_{0}(v, p(T))}+a_{1}^{4}(T)-6 a_{1}^{2}(T) a_{2}(T)+ \\
& +4 a_{1}(T) a_{3}(T)+3 a_{2}^{2}(T)
\end{aligned}
$$

However, in contradiction to [10], all the cumulants $a_{n}(T)$ are now functions of the temperature, since they contain a temperature-dependent parameter $p(T)(2.6)$. This should be borne in mind, but, for convenience, we will denote these coefficients as $a_{n}(T) \equiv a_{n}$. Due to the condition $p(T)>0$, the special functions $T_{n}(v, p)$ are rapidly convergent series

$T_{n}(v, p(T))=\sum_{m=0}^{\infty} \frac{v^{m}}{m !} m^{n} e^{-p(T) m^{2}}$.

Taking (2.7) and (2.6) into account, we now find a precise representation of the GPF of the model:

$$
\begin{aligned}
& \Xi=g_{W} e^{-\left(a_{0}+\frac{\beta \mu^{2}}{2 W(0)}\right) N_{v}} \int(d \tilde{t})^{N_{v}} \times \\
& \times \exp \left[\sqrt{N_{v}}\left(\frac{\mu}{W(0)}-a_{1}\right) \tilde{t}_{0}-\frac{1}{2} \sum_{\mathbf{k} \in \mathcal{B}_{c}} D(k) \tilde{t}_{\mathbf{k}} \tilde{t}_{-\mathbf{k}}-\right. \\
& \left.-\sum_{n=3}^{\infty} \frac{a_{n}}{n !} N_{v}^{\frac{2-n}{2}} \sum_{\substack{\mathbf{k}_{1}, \ldots, \mathbf{k}_{n} \\
\mathbf{k}_{i} \in \mathcal{B}_{c}}} \tilde{t}_{\mathbf{k}_{1}} \ldots \tilde{t}_{\mathbf{k}_{n}} \delta_{\mathbf{k}_{1}+\ldots+\mathbf{k}_{n}}\right]
\end{aligned}
$$

where we denoted

$D(k)=a_{2}+\frac{1}{W(k) \beta}$.

\section{An Approximate Calculation of the Grand Partition Function}

Expression (3.4) is comparable to the one obtained in $[8,10]$, but there is a crucial difference in the present case. This expression is true for any

$R_{0} / \alpha>4 \ln 2$

as well as for arbitrary values of the parameter $\chi>0$. Inequality (4.1) is peculiar for the description of alkali metals (particularly, Cs, Rb, K, Na, and so on) by the Morse potential [1, 4, 12].

It is impossible to calculate (3.4) in the general form, since there is an infinite power series in the variable $\tilde{t}_{\mathbf{k}}$ in the exponent. In connection with this, we use an approximation of the $\rho^{4}$-model, which consists in the cutting-off of the terms proportional to the fifth power of the variable $\tilde{t}_{\mathbf{k}}$ and more $\left(n_{0} \geq 5\right)$. In this case, (3.4) looks like the functional representation of the 3D Ising model in an external field and, respectively, belongs to the same universality class [9]. In our case, the chemical potential $\mu$ corresponds to an 
external field. In order to calculate GPF (3.4), we make the variable substitution defined by

$\tilde{t}_{\mathbf{k}}=\rho_{\mathbf{k}}+a_{34} \sqrt{N_{v}} \delta_{\mathbf{k}}, \quad a_{34}=-\frac{a_{3}}{a_{4}}$,

which is aimed to destroy the cubic terms of $\tilde{t}_{\mathbf{k}}$. As a result, we obtain the expression

$$
\begin{aligned}
& \Xi=g_{W} e^{N_{v}\left(E_{\mu}-a_{0}\right)} \int(d \rho)^{N_{v}} \times \\
& \times \exp \left[\sqrt{N_{v}} M \rho_{0}-\frac{1}{2} \sum_{\mathbf{k} \in \mathcal{B}_{c}} d(k) \rho_{\mathbf{k}} \rho_{-\mathbf{k}}-\right. \\
& \left.-\frac{a_{4}}{24} \frac{1}{N_{v}} \sum_{\substack{\mathbf{k}_{1}, \ldots, \mathbf{k}_{4} \\
\mathbf{k}_{i} \in \mathcal{B}_{c}}} \rho_{\mathbf{k}_{1}} \ldots \rho_{\mathbf{k}_{4}} \delta_{\mathbf{k}_{1}+\ldots+\mathbf{k}_{4}}\right],
\end{aligned}
$$

with the notations

$$
\begin{aligned}
& E_{\mu}=-\frac{\beta W(0)}{2}\left(M+\tilde{a}_{1}\right)^{2}+M a_{34}+\frac{d(0)}{2} a_{34}^{2}-\frac{a_{4}}{24} a_{34}^{4}, \\
& M=\frac{\mu}{W(0)}-\tilde{a}_{1}, \\
& \tilde{a}_{1}=a_{1}+d(0) a_{34}+\frac{a_{4}}{6} a_{34}^{3} .
\end{aligned}
$$

The coefficient $d(k)$ has the form

$d(k)=\frac{1}{\beta W(k)}-\tilde{a}_{2}, \quad \tilde{a}_{2}=\frac{a_{4}}{2} a_{34}^{2}-a_{2}$.

On this stage, we use a type of the mean-field approximation considering only the variables $\rho_{\mathbf{k}}$ with $\mathbf{k}=0$ (see [10]). Applying this approximation, one would describe a behavior of the model in a wide range of temperatures (excluding a narrow vicinity of the critical point, where the contribution of the variables $\rho_{\mathbf{k}}$ with $\mathbf{k} \neq 0$ is important).

In this approximation, the GPF has the form

$$
\Xi \simeq g_{W}^{\prime} e^{N_{v} E_{\mu}} \int_{-\infty}^{\infty} d \rho_{0} \exp \left[N_{v} E\left(\rho_{0}\right)\right] .
$$

We obtained $E\left(\rho_{0}\right)$, by using the change of the variables $\rho_{0}^{\prime}=\sqrt{N_{v}} \rho_{0}$

$E\left(\rho_{0}\right)=M \rho_{0}-\frac{1}{2} d(0) \rho_{0}^{2}-\frac{a_{4}}{24} \rho_{0}^{4}$.

In the mean-field approximation [5], the transition temperature can be determined from the following condition:

$d(0)=\frac{1}{\beta_{c} W(0)}-\tilde{a}_{2 c}=0$,

\section{8}

where the index $c$ means that the value is taken at a fixed temperature $T_{c}: a_{n c}=a_{n}\left(T_{c}\right), W(0)=$ $=\left.W(k)\right|_{\mathbf{k}=0}$. Expression (4.7) gives the definition of the critical temperature

$k_{\mathrm{B}} T_{c}=\tilde{a}_{2 c} W(0)$.

Taking (2.4) into account, we get

$d(0)=\tilde{a}_{2 c}(\tau+1)-\tilde{a}_{2}, \tau=\frac{\left(T-T_{c}\right)}{T_{c}}$.

Using the Laplace method [3], we obtain the asymptotic form of the GPF as follows:

$\Xi \simeq g_{W}^{\prime} \exp \left[N_{v} E_{\mu}+N_{v} E\left(\bar{\rho}_{0}\right)\right]$

where the value of $\rho_{0}=\bar{\rho}_{0}$ corresponds to the maximum of $E\left(\rho_{0}\right)$. Having an explicit expression of GPF (4.10), we can find an equation for the average density of the system, by using the well-known formula

$\bar{n}=\frac{1}{N_{v}} \frac{\partial \ln \Xi}{\partial \beta \mu}=\frac{\partial E_{\mu}}{\partial \beta \mu}+\frac{\partial E_{0}\left(\bar{\rho}_{0}\right)}{\partial \beta \mu}$.

In view of (4.11), we have

$\bar{n}=n_{c}-M-\frac{\bar{\rho}_{0}}{\beta W(0)}$,

where

$n_{c}=-a_{1}-a_{2} a_{34}+\frac{a_{4}}{3} a_{34}^{3}$,

Taking (4.8) into account, the following equality is self-evident:

$\beta W(0)=\frac{1}{\tilde{a}_{2 c}(\tau+1)}$.

Since $\bar{\rho}_{0}=\bar{\rho}_{0}(\tau, M)$, the equality (4.12) is the key expression in the framework of the grand canonical ensemble. The condition of maximum for $E\left(\bar{\rho}_{0}\right)$,

$M=d(0) \bar{\rho}_{0}+\frac{a_{4}}{6} \bar{\rho}_{0}^{3}$,

provides an equation connecting the chemical potential $M$ and the density of the system $\bar{n}$ :

$m^{3}+m p_{b}+q_{b}=0$,

where

$m=M+\left(\bar{n}-n_{c}\right)$,

$p_{b}=\frac{6}{a_{4}}\left[\tilde{a}_{2 c}(\tau+1)\right]^{2} \tilde{a}_{2}$,

ISSN 2071-0194. Ukr. J. Phys. 2017. Vol. 62, No. 10 
$q_{b}=-\frac{6}{a_{4}}\left[\tilde{a}_{2 c}(\tau+1)\right]^{3}\left(\bar{n}-n_{c}\right)$.

Solutions of this equation are plotted in Fig. 1. Obviously, curve 1 is the one that shows a physical dependence (namely, the growth of $m_{n}$ with the density). A solution corresponding to curve 1 exists on the density interval

$n_{\min } \leqslant \bar{n} \leqslant n_{\max }$

which is compatible with a particular range of values of $m_{1}$. The chemical potential as a function of the density has the form

$$
\begin{aligned}
& M(\bar{n})=m_{1}(\bar{n})-\left(\bar{n}-n_{c}\right), \\
& m_{1}(\bar{n})=2 \sqrt{\frac{2 \tilde{a}_{2}^{3}}{a_{4}}} \sin \frac{\alpha_{b}(\bar{n})}{3}, \\
& \alpha_{b}(\bar{n})=\arcsin \left[\sqrt{\frac{9 a_{4}}{8 \tilde{a}_{2}^{3}}}\left(\bar{n}-n_{c}\right)\right] .
\end{aligned}
$$

Based on the data of computer experiments [15] for sodium and potassium, we consider $n_{\min }=0.1$. Consequently, there is the equation

$n_{c}=\frac{2}{3}\left[\frac{2 \tilde{a}_{2}^{3}}{a_{4}}\right]^{1 / 2}-0.1$,

by means of which we find the parameter $v$ as a function of the temperature. The change of the density from 0.1 to the limit value $n_{\max }$ is equivalent to the increase of the chemical potential from $M_{\min }$ to $M_{\max }$.

Note that the solution $m_{1}$ (4.18) (Fig. 1) is equitable for some (bounded) range of chemical potential values $M=f(\bar{n}, \tau)$ (see Fig. 2 )

\section{Description of the First-Order Phase Transitions}

According to the well-known formula $P V=k_{\mathrm{B}} T \ln \Xi$, the equation of state of the cell fluid model can be written in the form

$\frac{P v}{k_{\mathrm{B}} T}=\frac{\ln g_{W}^{\prime}}{N_{v}}+E_{\mu}+M(\bar{n}) \bar{\rho}_{0 i}-\frac{1}{2} \tilde{d}(0) \bar{\rho}_{0 i}^{2}-\frac{a_{4}}{24} \bar{\rho}_{0 i}^{4}$,

where $E_{\mu}$ is defined in (4.3), and the quantities $\bar{\rho}_{0 i}$ $i=1,2,3$ are solutions of the equation

$$
\begin{aligned}
& \bar{\rho}_{0 i}^{3}+p_{Q} \bar{\rho}_{0 i}+q_{Q}=0, \\
& p_{Q}=\frac{6 d(0)}{a_{4}} q_{Q}=-\frac{6 M(\bar{n})}{a_{4}} .
\end{aligned}
$$

ISSN 2071-0194. Ukr. J. Phys. 2017. Vol. 62, No. 10

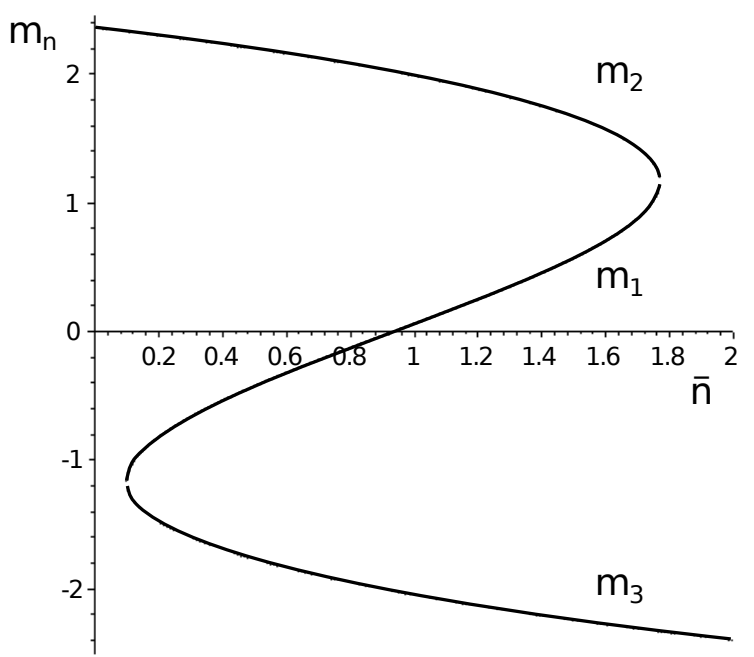

Fig. 1. Plot of the solutions $m_{n}$ as functions of the number density $\bar{n}$

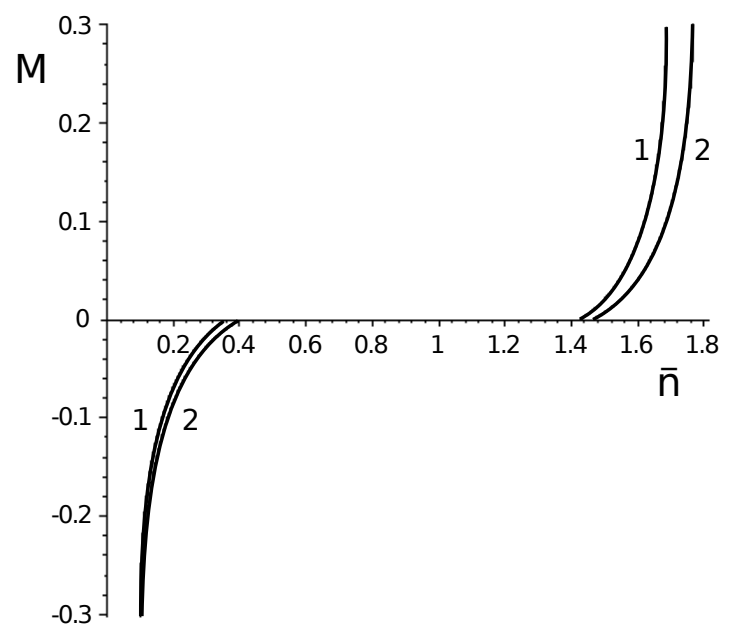

Fig. 2. Plot of the chemical potential $M(\bar{n})$ as a function of the number density $\bar{n}$ (curve 1 is for potassium, curve 2 is for sodium)

At $T>T_{c}$, the discriminant of Eq. (5.2)

$Q=\left(\frac{2 d(0)}{a_{4}}\right)^{3}+\left(-\frac{3 M}{a_{4}}\right)^{2}$

is positive, since $p_{Q}>0$. So, we have a single real solution of (5.2). This solution can be found directly from Eq. (5.2) as

$\bar{\rho}_{0 b}=\left(\frac{3 M(\bar{n})}{a_{4}}+\sqrt{Q}\right)^{1 / 3}+\left(\frac{3 M(\bar{n})}{a_{4}}-\sqrt{Q}\right)^{1 / 3}$. 

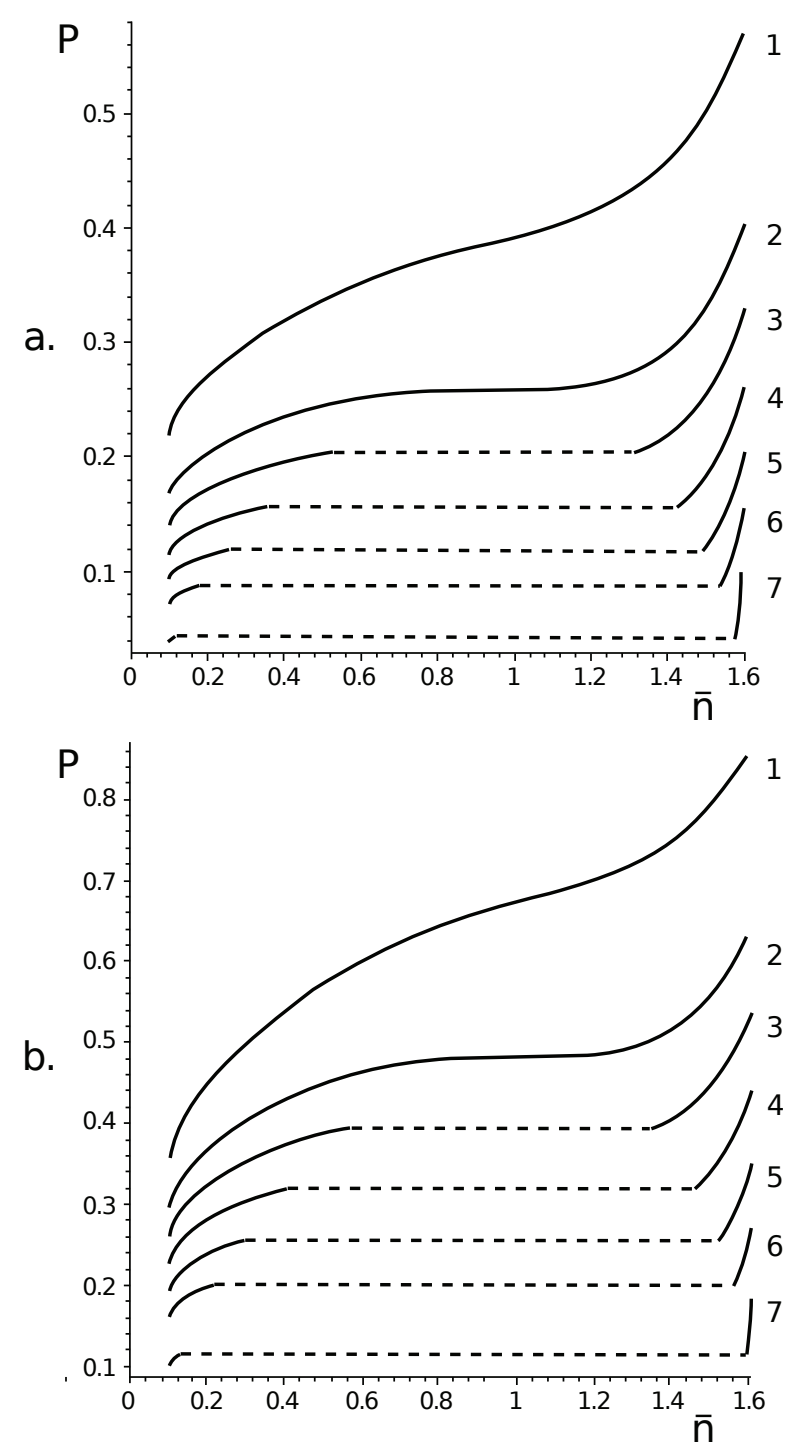

Fig. 3. Plots of the pressure $P(\bar{n})$ as a function of the number density $\bar{n}$ at different temperatures: curve 1 is for $\tau=0.1$, curve 2 is for $\tau=0$, curve 3 is for $\tau=-0.05$, curve 4 is for $\tau=-0.1$, curve 5 is for $\tau=-0.15$, curve 6 is for $\tau=-0.2$, and curve 7 is for $\tau=-0.3$. Data for potassium $a$, data for sodium $b$

For $T>T_{c}$, the equation of state or pressure as a function of the temperature and the density takes the form

$$
\begin{aligned}
& \frac{P}{k_{\mathrm{B}} T_{c}}=\frac{\tau+1}{v(T)}\left(f+\frac{M(\bar{n})}{2 \tilde{a}_{2 c}(\tau+1)}[M(\bar{n})+2 \bar{n}]-\right. \\
& \left.-\frac{d(0) m_{1}^{2}(\bar{n})}{2\left[\tilde{a}_{2 c}(\tau+1)\right]^{2}}-\frac{a_{4}}{24} \frac{m_{1}^{4}(\bar{n})}{\left[\tilde{a}_{2 c}(\tau+1)\right]^{4}}\right) .
\end{aligned}
$$

$f=\frac{1}{N_{v}} \ln g_{W}^{\prime}-a_{0}+\frac{d(0)}{2} a_{34}^{2}-\frac{\tilde{a}_{1}^{2}}{2 \tilde{a}_{2 c}(\tau+1)}-\frac{a_{4}}{24} a_{34}^{4}$.

The explicit expression of the pressure as a function of the density at the critical temperature deduced from (5.5) by substituting $T_{c}$ for $T$ is as follows:

$$
\begin{aligned}
& \frac{\left.P v\right|_{T=T_{c}}}{k_{\mathrm{B}} T_{c}}=f_{c}+\frac{M_{0}(\bar{n})}{2 \tilde{a}_{2 c}}\left[M_{0}(\bar{n})+\left.2 \bar{n}\right|_{T=T_{c}}\right]- \\
& -\frac{a_{4 c}}{24}\left[\frac{\left.m_{1}(\bar{n})\right|_{T=T_{c}}}{\tilde{a}_{2 c}}\right]^{4}, \\
& f_{c}=\frac{1}{N_{v}} \ln g_{W}^{\prime}-a_{0 c}+\frac{a_{4 c}}{24} a_{34 c}^{4}-\frac{\tilde{a}_{1 c}^{2}}{2 \tilde{a}_{2 c}} .
\end{aligned}
$$

The total chemical potential $M_{0}$ as a function of the density is the following:

$$
M_{0}=\left.m_{1}(\bar{n})\right|_{T=T_{c}}-\left(\left.\bar{n}\right|_{T=T_{c}}-n_{g c}\right) .
$$

Indices 0 and $c$ denote that $M_{0}$ and $a_{n c}$ correspond to the case of $T=T_{c}$. The plots of the pressure $P=P(\bar{n})$ vs the average density expressed by (5.5) (curve 1) and $\left.P\right|_{T=T_{c}}=\left.P\right|_{T=T_{c}}(\bar{n})$ expressed by (5.6) (curve 2) are shown in Fig. 3 for sodium $(a)$ and potassium $(b)$.

At $T<T_{c}$, we have three real solutions of (5.2):

$$
\begin{aligned}
& \bar{\rho}_{01}=2 \rho_{0 r} \cos \frac{\alpha_{m}}{3}, \\
& \bar{\rho}_{02}=-2 \rho_{0 r} \cos \left(\frac{\alpha_{m}+\pi}{3}\right), \\
& \bar{\rho}_{03}=-2 \rho_{0 r} \cos \left(\frac{\alpha_{m}-\pi}{3}\right),
\end{aligned}
$$

where

$\rho_{0 r}=\left(-2 d(0) / a_{4}\right)^{1 / 2}$,

and the angle $\alpha_{m}$

$\alpha_{m}=\arccos \frac{M}{M_{q}}, \quad M_{q}=\sqrt{-\frac{8[d(0)]^{3}}{9 a_{4}}}$.

The solution $\rho_{01}$ fits the stability condition in the interval $M \in\left[0, M_{\max }\right]$, as well as $\rho_{03}-$ in $M \in$ $\in\left[M_{\min }, 0\right]$ (see Fig. 4).

As a result, we can present the equation of state as

$\frac{P}{k_{\mathrm{B}} T_{c}}=-\frac{\tau+1}{v(T)}\left(\frac{1}{N_{v}} \ln g_{W}^{\prime}+E_{\mu}(\bar{n})+E_{1}\left(\bar{\rho}_{03}\right) \times\right.$

ISSN 2071-0194. Ukr. J. Phys. 2017. Vol. 62, No. 10 


$$
\begin{aligned}
& \times \Theta\left(\bar{n}_{12}-\bar{n}\right)+E_{2}\left(\bar{\rho}_{03}\right) \Theta\left(\bar{n}-\bar{n}_{12}\right) \Theta\left(-\bar{n}+\bar{n}_{20}\right)+ \\
& \left.+E_{3}\left(\bar{\rho}_{01}\right) \Theta\left(\bar{n}-\bar{n}_{03}\right) \Theta\left(\bar{n}_{34}-\bar{n}\right)+E_{4}\left(\bar{\rho}_{01}\right) \Theta\left(\bar{n}-\bar{n}_{34}\right)\right) .
\end{aligned}
$$

The quantity $E_{\mu}$ is defined by formula (4.3). The functions $E_{n}\left(\bar{\rho}_{0}\right)$ have the form

$E_{n}\left(\bar{\rho}_{0 n}\right)=M(\bar{n}) \bar{\rho}_{0 n}-\frac{d(0)}{2} \bar{\rho}_{0 n}^{2}-\frac{a_{4}}{24} \bar{\rho}_{0 n}^{4}$,

where the notation $\bar{\rho}_{0 n}$ is either $\bar{\rho}_{01}$ from (5.7) for $E_{3}\left(\bar{\rho}_{01}\right)$ and $E_{4}\left(\bar{\rho}_{01}\right)$, or $\bar{\rho}_{03}$ from $(5.7)$ for $E_{1}\left(\bar{\rho}_{03}\right)$ and $E_{2}\left(\bar{\rho}_{03}\right)$. Equation (5.10) also includes the values of densities: $\bar{n}_{12}$ is $\left(M=-M_{q}\right)$

$\bar{n}_{12}=n_{c}-2 \tilde{a}_{2} \rho_{0 r}+4 \frac{a_{4}}{3} \rho_{0 r}^{3}$,

$\bar{n}_{34}$ is $\left(M=M_{q}\right)$

$\bar{n}_{34}=n_{c}+2 \tilde{a}_{2} \rho_{0 r}-4 \frac{a_{4}}{3} \rho_{0 r}^{3}$,

$\bar{n}_{20}$ and $\bar{n}_{03}$ are the densities of a liquid-vapor transition

$\bar{n}_{20}=n_{c}-\sqrt{3} \tilde{a}_{2 c}(\tau+1) \rho_{0 r}$,
$\bar{n}_{03}=n_{c}+\sqrt{3} \tilde{a}_{2 c}(\tau+1) \rho_{0 r}$.

\section{Analytic Results}

As we mentioned before, the liquid-vapor coexistence curves for $\mathrm{Na}$ and $\mathrm{K}$ have already been calculated in [15] by the Monte Carlo simulation in the grand canonical ensemble. Therefore, we can compare them with our theoretical results. To do it, we calculated the binodals in the same temperature interval, as in [15]. The results of this comparison are presented in Fig. 5 (using the reduced units $T / T_{c}$ and $\bar{n} / \bar{n}_{c}$ ). Both the gas branches of our binodals and those from the simulation data follow the same trend. The agreement is unsatisfactory for the liquid branches. The critical point coordinates for sodium and potassium obtained in [15] are

$\rho_{c}^{*}(\mathrm{Na})=1.430, \quad T_{c}^{*}(\mathrm{Na})=5.874$,

$\rho_{c}^{*}(\mathrm{~K})=1.125, \quad T_{c}^{*}(\mathrm{~K})=5.05$,

(in reduced units $T^{*}=k_{\mathrm{B}} T / D$ and $\rho^{*}=\rho / R_{0}^{3}$ ). Our results give the following values:

$\bar{n}_{c}(\mathrm{Na})=0.997, \quad T_{c}(\mathrm{Na})=5.760$,

$\bar{n}_{c}(\mathrm{~K})=0.935, \quad T_{c}(\mathrm{~K})=5.037$,

ISSN 2071-0194. Ukr. J. Phys. 2017. Vol. 62, No. 10

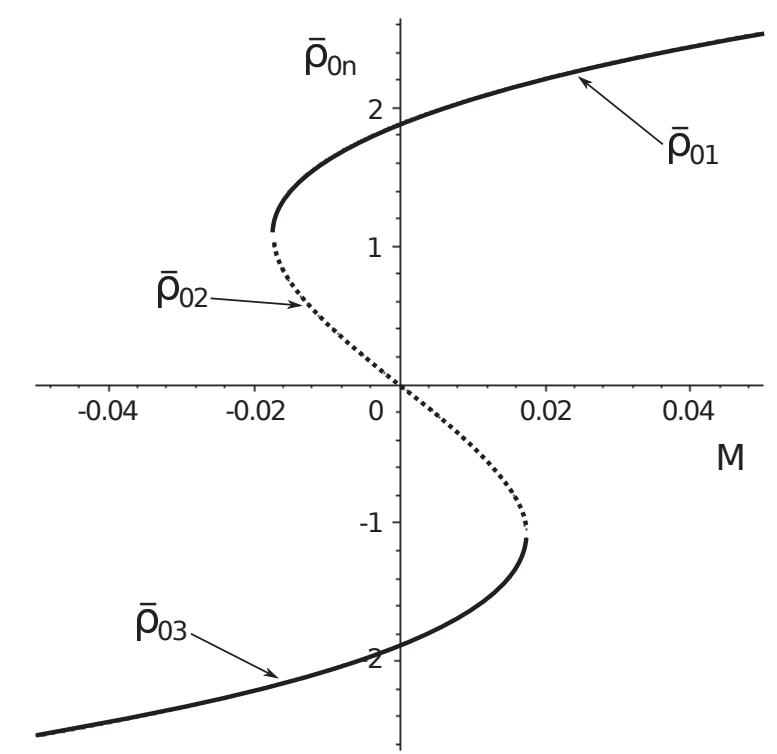

Fig. 4. Plots of the solutions $\bar{\rho}_{0} n$ as functions of the effective chemical potential $M$

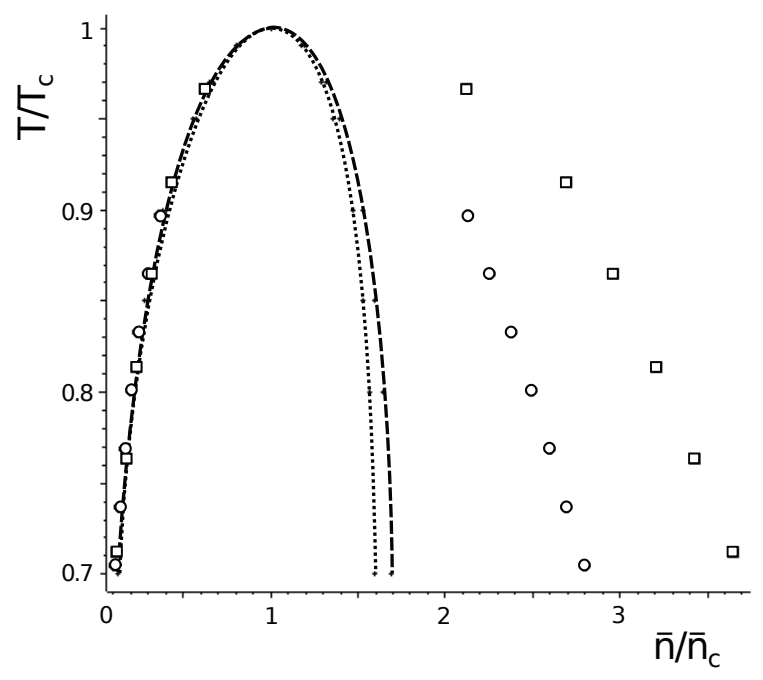

Fig. 5. Coexistence curves: analytic results for $\mathrm{K}-$ doted curve, $\mathrm{Na}$ - dashed curve; simulation data [15]: $\mathrm{K}$ - rings, $\mathrm{Na}$ - boxes,

using the corresponding values of parameters of the model

$\chi=1.124,\left.\quad v\right|_{T_{c}}=2.419$ for $\mathrm{Na}$,

$\chi=1.198,\left.\quad v\right|_{T_{c}}=2.940$ for $\mathrm{K}$,

according to $(2.6) p(\mathrm{Na})=1.81$ and $p(\mathrm{~K})=2.01$.

As one can see, the estimated $\mathrm{Na}$ and $\mathrm{K}$ critical temperatures are close to the simulations values. This

871 
is, however, not true for the critical densities of both substances, where the analytically obtained critical density is lower than the value from computer experiments. Nevertheless, the critical density of sodium is higher in both cases than the value for potassium.

\section{Discussion and Conclusions}

A theoretical description of the first-order phase transition in alkali metals is proposed. The interaction of this type of metals is known to be well described by the Morse potential. The critical density and critical temperature of potassium and sodium are calculated, by using numerical results for such a potential [12] both with particular values of microscopic parameters. We have obtained a quite good agreement with computer simulation data, despite applying a type of the mean-field approximation. The equation of state is calculated. At the region above the critical temperature, the isotherms of pressure behave themselves as smooth increasing functions. There is a gas-liquid phase transition below the critical temperature. It is important that, in the proposed approach, there is no need to use the Maxwell construction. In contradistinction to other approaches connected to the mean-field approximation (e.g., the van der Waals theory), a plateau of the pressure, which depicts a transition from gas to a liquid state, naturally arises as a result of calculations. This is achieved by applying the Laplace method to the calculation of the grand partition function in the $\rho^{4}$-model approximation. Although the method is approximate, we have obtained a good agreement with simulation data for the coexistence curves of sodium and potassium in the region of low densities, without using any phenomenological parameters.

The introduction of the parameter $\chi$ lies at the heart of the method. This parameter is necessary in order to take a particular part of the interaction potential $\chi \Psi(0)$, which is used to calculate the Jacobian of the transition from variables in the direct space to the collective variables. A value of the critical temperature of the model depends on $\chi$. For this reason, we choose a value of this parameter so that we obtain values of $T_{c}$ (for particular substances), which correspond to the data of computer experiment. Note that, according to formula (2.6), $\chi$ determines the parameter $p$. The last parameter $v$ appears as a result of choosing a cell fluid model. Recall that $v$ is the vol- ume of a cell in $R_{0}$-units. Due to the self-consistent calculation, one gets values of this parameter from the condition (4.19).

The plot of binodals in Fig. 5 shows that, unfortunately, our approach does not give quantitatively satisfactory results in the fluid region. As an option, a somewhat better description can be achieved by introducing phenomenological parameters. Something similar was done in [2], where the authors got good results for fluids with different interaction potentials. On the other hand, using approximations of a higher power in $\rho$ might be helpful. Taking the particular results $[11,13]$ into account, we conclude that the appliance of $\rho^{m}$-models with $m>4$ stipulates an asymmetry of the coexistence curve in the density region for liquids.

This work was partly supported by the European Commission under the project STREVCOMS PIRSES-2013-612669, FP' EU IRSES projects No. 612707 (DIONICOS).

1. J.D. Bringas, J. Lopez-Lemus, B. Ibarra-Tandi, P. Orea. Liquid-vapour interface of simple fluids interacting by the modified Morse potential. Molecular Simulation 37, 449 (2011).

2. L.A. Bulavin, V.L. Kulinskii. Generalized principle of corresponding states and the scale invariant mean-field approach. J. Chem. Phys. 133, 134101 (2010).

3. M.V. Fedoryuk. Asymptotic methods in analysis. In Analysis I: Integral Representations and Asymptotic Methods. Encyclopaedia of Mathematical Sciences 13, eds. M.A. Evgrafov, R.V. Gamkrelidze (Springer, 1989) p. 83.

4. I.A. Girifalko, V.G. Weizer. Application of the Morse potential function to cubic metals. Phys. Rev. 114, 687 (1959).

5. L.P. Kadanoff. More is the same; Phase transitions and mean field theories. J. Stat. Phys. 137, 777 (2009).

6. Yu. Kozitsky, M. Kozlovskii. A phase transition in a continuum Curie-Weiss system with binary interactions. arXiv:1610.01845 [math-ph], (2016).

7. M.P. Kozlovskii, O.A. Dobush, R.V. Romanik. Concerning a calculation of the grand partition function of a fluid model. Ukr. J. Phys. 60, 805 (2015).

8. M. Kozlovskii, O. Dobush. Representation of the grand partition function of the cell model: The state equation in the mean-field approximation. J. Mol. Liq. 215, 58 (2016).

9. M.P. Kozlovskii. Free energy of 3D Ising-like system near the phase transition point. Cond. Mat. Phys. 12, 151 (2009).

10. M.P. Kozlovskii, O.A. Dobush. Phase transition in a cell fluid model. Condens. Matter Phys. 20, 23501 (2017).

11. V.L. Kulinskii, N.P. Malomuzh. The nature of the rectilinear diameter singularity. Physica A 388, 621 (2009).

ISSN 2071-0194. Ukr. J. Phys. 2017. Vol. 62, No. 10 
12. R.C. Lincoln, K.M. Koliwad. Morse-potential evaluation of second- and third-order elastic constants of some cubic metals. Phys. Rev. 157, 463 (1967).

13. J.F. Nicoll. Critical phenomena of fluids: Asymmetric Landau-Ginzburg-Wilson model. Phys. Rev. A 24, 2203 (1982).

14. A.L. Rebenko. Cell gas model of classical statistical systems Rev. Math. Phys. 25, 1330006 (2013).

15. J.K. Singh, J. Adhikari, S.K. Kwak. Vapor-liquid phase coexistence curves for Morse fluids. Fluid Phase Equilibria 248, 1 (2006).

16. I.R. Yukhnovskii. The phase transition of the first order in the critical region of the gas-liquid system. Cond. Mat. Phys. 17, 43001 (2014).

Received 30.07.17
М.П. Козловсъкий, О.А. Добуш, І.В. Пилюк

ВИКОРИСТАННЯ МОДЕЛІ КОМІРКОВӦ̈ РІДИНИ ДЛЯ ОПИСУ ФАЗОВОГО ПЕРЕХОДУ В ПРОСТИХ РІДКИХ ЛУЖНИХ МЕТАЛАХ

$\mathrm{P}$ е $з$ ю м е

Ця стаття охоплює теоретичний опис фазового переходу першого роду в рідких металах з використанням моделі коміркового плину. Результати отримано шляхом розрахунку великої статистичної суми без використання феноменологічних параметрів. Потенціал Морзе використовується для обчислення рівняння стану та кривої співіснування. Отримано конкретні результати для натрію та калію. Представлено порівняння результатів аналітичних виразів $з$ даними комп'ютерного моделювання. 